DOI: $10.4274 /$ jarem.galenos.2020.3640

J Acad Res Med 2021;11(1):117-119

\title{
Relapse of T-Cell Lymphoma as Isolated Brachial Plexus Neurolymphomatosis: A Case Report
}

\author{
(10) Kenan Kıbıcı1, (10) Berrin Erok2, (1) Ali Önder Atça 3, (1) Gülçin Yeğen4 \\ ${ }^{1}$ Altınbaş University Faculty of Medicine, Bahçelievler Medical Park Hospital, Department of Neurosurgery, İstanbul, Turkey \\ 2University of Health Sciences Turkey, Prof. Dr. Cemil Taşcıoğlu City Hospital, Clinic of Radiology, İstanbul, Turkey \\ ${ }^{3}$ Altınbaş University Faculty of Medicine, Bahçelievler Medical Park Hospital, Department of Radiology, İstanbul, Turkey \\ ${ }^{4}$ istanbul University Faculty of Medicine, Department of Pathology, İstanbul, Turkey
}

Cite this article as: Kıbıcı K, Erok B, Atça AÖ, Yeğen G. Relapse of T-Cell Lymphoma as Isolated Brachial Plexus Neurolymphomatosis: A Case Report. J Acad Res Med 2021;11(1):117-119

\begin{abstract}
Neurolymphomatosis (NL) is the neoplastic endoneurial invasion of the peripheral nervous system. It is a rare and challenging diagnosis, but it should be taken into account in the differential diagnosis of peripheral neuropathy, particularly in patients with a documented history of haematologic malignancy. Magnetic resonance neurography is very useful in diagnosis and especially when correlated with positron emission tomography/ computed tomography (PET/CT). In equivocal cases, nerve biopsy can be considered when the benefit outweighs the risk. We aimed to report a case of a 30-year-old male patient who was in complete remission from T-lymphoblastic lymphoma, presenting with clinical findings indicating initially ulnar entrapment. However, with the demonstration of brachial plexopathy with axonal loss in electrodiagnostic studies, magnetic resonance imaging neurography dedicated to brachial plexus was carried out and revealed pathological enhancement associated with mild fluorodeoxyglucose (FDG) uptake on PET/CT. Moreover, NL, due to the relapse of T-cell lymphoma, was diagnosed through incisional biopsy, showing diffuse infiltration of blast cells positive for terminal deoxynucleotidyl transferase, CD3 and CD10. Further, radiotherapy and systemic chemotherapy were initiated, and symptoms recovered with regression of pathological FDG uptake.
\end{abstract}

Keywords: Neurolymphomatosis, T-cell lymphoblastic lymphoma, brachial plexopathy

\section{INTRODUCTION}

Neurolymphomatosis (NL) is the neoplastic endoneurial invasion of the peripheral nervous system (PNS). It differs from meningeal lymphomatosis, which is the infiltration of the PNS due to subarachnoid seeding (1). Furthermore, NL occurs more frequently with the spread of a known malignancy into the PNS from systemic sites or from the primary central nervous system (CNS) lymphoma (PCNSL) as a progression in the course of the disease or as a relapse following complete remission. More rarely, it may occur as an initial manifestation of the disease. We report a case of a 30-year-old male patient with right brachial plexopathy caused by the relapse of T-lymphoblastic lymphoma.

\section{CASE PRESENTATION}

A 30-year-old male patient presented with right medial forearm and hand pain and numbing in the fourth and fifth fingers, initially indicating ulnar nerve entrapment. A year ago, he was treated for T-lymphoblastic leukaemia/lymphoma and achieved complete remission with no pathological fluorodeoxyglucose

ORCID IDs of the authors: K.K. 0000-0002-5912-9648; B.E. 0000-0001-8036-547X; A.Ö.A. 0000-0002-7500-3316; G.Y. 0000-0003-2497-219X.

Corresponding Author/Sorumlu Yazar: Berrin Erok, E-mail: drberrinerok@hotmail.com
Received Date/Geliş Tarihi: 29.07.2020 Accepted Date/Kabul Tarihi: 22.12.2020

CC Copyright 2021 by University of Health Sciences Turkey, Gaziosmanpaşa Training and Research Hospital. Available on-line at www.jarem.org 
(FDG) uptake in positron emission tomography/computed tomography (PET/CT). In neurological examination, muscular strengths of the forearm flexors and intrinsic hand muscles were poor, and there was pain-related paraesthesia. Moreover, no disc impingement was demonstrated by cervical magnetic resonance imaging (MRI). Ulnar nerve entrapment was considered; however, electrodiagnostic studies revealed right lower truncus brachial plexopathy associated with axonal loss and active denervation. Gadolinium-enhanced MRI neurography showed a 25×32×41 mmsized enhancing extrapulmonary lesion located at the apical part of the right hemithorax, affecting the middle and lower part of the brachial plexus (BP) (Figure 1). No pathological signal was detected in the adjacent pulmonary parenchyma. Also, there was no extension into the spinal canal. PET/CT showed a mild hypermetabolic FDG uptake (maximum standardized uptake value: 3.04$)$ in the lesion. Moreover, NL was considered and incisional biopsy was performed to make an exact diagnosis. During the antigenic assessment, diffuse infiltration of blast cells positive for terminal deoxynucleotidyl transferase, CD3 and CD10 was demonstrated (Figure 2). Once again, radiotherapy and systemic chemotherapy was started. With the regression of pathological FDG uptake in PET/CT, his symptoms recovered with improvement of muscle strengths from $3 / 5$ to $5 / 5$.

Written informed consent has been taken from the patient.

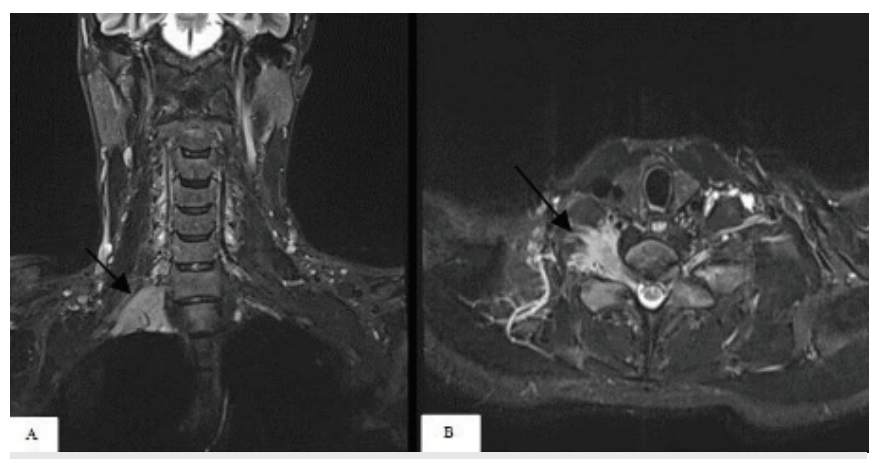

Figure 1. Brachial plexus magnetic resonance imaging neurography with gadolinium, short-tau inversion recovery sequence sagittal (A) and axial (B) images showing pathologic enhancement in the right brachial plexus (black arrows)

\section{DISCUSSION}

$\mathrm{NL}$ is a very rare neurological manifestation of haematologic malignancies that should be distinguished from the more prevalent causes of PNS involvement, such as radiation-induced peripheral neuropathy or paraneoplastic syndromes. Most cases of NL are associated with diffuse large B-cell non-Hodgkin's lymphoma (DLBCL) but can also occur in T-cell and NK-cell lymphomas or acute leukaemias. According to the report of the International Primary CNS Lymphoma Collaborative Group, NL developed in 50 patients over a 16-year period, of which $90 \%$ were associated with non-Hodgkin's lymphoma (NHL) and 10\%

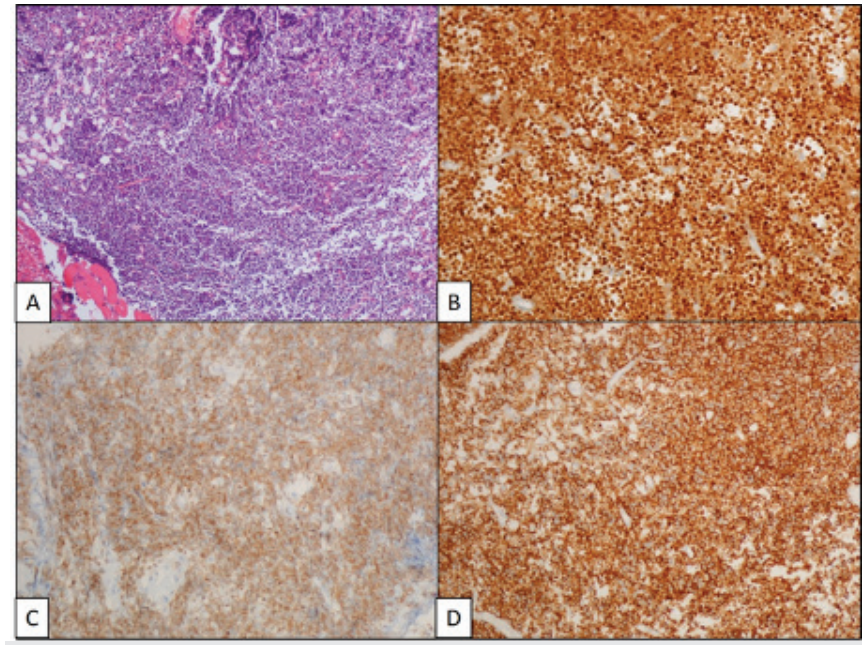

Figure 2. Incisional biopsy of the brachial plexus lesion showing diffuse infiltration of blast cells (H\&E) (A), which were positive for terminal deoxynucleotidyl transferase (B), CD3 (C) and CD10 (D) in the antigenic assessment

with acute leukaemia (2). In a 12-year retrospective analysis of 1,181 patients with NHL, 22 cases of NL were identified, of which 20 patients were associated with DLBCL, 1 patient with mantle cell lymphoma, and 1 patient with peripheral T-cell lymphoma (3). In another report of 23 cases with NL of lumbosacral plexus, $\mathrm{B}$-cell histology was seen in 21 cases and T-cell histology in only 2 cases (4). In our patient, NL was related to a relapse of T-cell lymphoma. Since the clinical findings are not clear, it is frequently misdiagnosed, especially when the neuropathy is the initial presentation or manifests after a period of complete remission, as in our case. When the BP is affected, the presenting symptoms are weakness, paraesthesia and pain radiating down to the upper limb or localised in the forearm and hand in cases of middle and lower trunk involvement, as in our case. In contrast to the radiation-induced brachial plexopathy that commonly affects the upper trunk or metastatic brachial plexopathy that has a predilection for the lower trunk, all parts of BP may be invaded by lymphomatous cells from the roots to the distal branches with no distinctive distribution (5). As suggestive of $N L$, severe pain, rapid evolution and asymmetric distribution have been reported (2). However, relatively painless or symmetrical neuropathy has also been defined $(6,7)$. Although axonal sensory motor neuropathy is more frequent in nerve conduction studies, pure demyelinating and mixed neuropathy have also been reported (7). Moreover, due to its low sensitivity, cerebrospinal fluid (CSF) cytology is used in the diagnosis but is not always helpful (6). Neuroimaging studies with MRI neurography often provide a correct diagnosis, especially when performed together with PET/CT. On MRI, normal nerves are usually smaller than the accompanying arteries, showing gradual tapering in a well-organised fascicular distribution with isointensity to skeletal muscles on both T1w and T2w images without enhancement, except the dorsal root ganglia which should not be confused with a tumoral process. In $\mathrm{NL}$, focal or diffuse nerve enlargement, fascicular disorganisation, 
hyperintensity on T2w or short-tau inversion recovery sequences and significant focal or diffuse gadolinium enhancement are seen. Although these are not specific for NL, they are highly suggestive when associated with PET/CT, which is the most accurate modality in the evaluation of nodal and extranodal spread of lymphoma $(8,9)$. In nerve pathology, the gold standard method of diagnosis is nerve biopsy, but it is not performed in all patients because of a significant risk of permanent nerve damage. Moreover, it is concerned when the benefit outweighs the risk and the imaging studies along with CSF examinations are inconclusive. In general, treatment of NL is similar to that of PCNSL, which includes systemic and/or intrathecal chemotherapy with or without radiotherapy (8). In rare instances, surgical intervention, such as emergent decompression, is needed. Our patient was treated with radiotherapy and systemic chemotherapy with achievement of complete remission. Since the relapse of the lymphoma in our patient was localised to the right $\mathrm{BP}$, radiotherapy was performed with limited-field radiation. Despite the treatment, the prognosis is worse in patients with NL than in those without (10). Although the lack of optimal treatment one of the causes, it may also be due to misdiagnosis that leads to delayed targeted treatment.

\section{CONCLUSION}

$\mathrm{NL}$ remains a rare and challenging diagnosis. It should be considered when a patient with a known history of haematologic malignancy presents with peripheral neuropathy, especially when the symptoms are asymmetric and rapidly evolving. MRI neurography and PET/CT are very helpful and should be used together to improve sensitivity. In equivocal cases, nerve biopsy can be used for early diagnosis when the benefit outweighs the risk, especially when, as in our case, there is an already existing axonal pattern of damage.
Informed Consent: Written informed consent has been taken from the patient.

Peer-review: Externally and internally peer-reviewed.

Author Contributions: Surgical and Medical Practices - K.K.; Concept K.K., B.E., A.Ö.A., G.Y.; Design - B.E., A.Ö.A.; Data Collection or Processing - B.E.; Analysis or Interpretation - K.K., B.E. G.Y.; Literature Search - B.E.; Writing - K.K., B.E.

Conflict of Interest: The authors have no conflict of interest to declare.

Financial Disclosure: The authors declared that this study has received no financial support.

\section{REFERENCES}

1. Stark, AM, Mehdorn, HM. Leptomeningeal metastasis of an intradural malignant peripheral nerve sheath tumor. J Clin Neurosci 2013; 20: 11813.

2. Grisariu S, Avni B, Batchelor TT, van den Bent MJ, Bokstein F, Schiff D, et al. Neurolymphomatosis: an International Primary CNS Lymphoma Collaborative Group report. Blood 2010; 115: 5005-11.

3. Narita K, Terao T, Tsushima T, Kitadate A, Miura D, Takeuchi M, et al. Clinical features, diagnosis, and prognosis of 22 cases of neurolymphomatosis: a single-Institution experience over 12 years. Blood 2019; 134(Suppl 1): 1630.

4. Bourque PR, Sampaio ML, Warman-Chardon J, Samaan S, Torres C. Neurolymphomatosis of the lumbosacral plexus and its branches: case series and literature review. BMC Cancer 2019; 19: 1149.

5. Khadilkar SV, Khade SS. Brachial plexopathy. Ann Indian Acad Neurol 2013; 16: 12-8.

6. Baehring JM, Damek D, Martin EC, Betensky RA, Hochberg FH. Neurolymphomatosis. Neuro Oncol 2003; 51: 104-15.

7. Shree R, Goyal MK, Modi M, Gaspar BL, Radotra BD, Ahuja CK, et al. The diagnostic dilemma of NL. J Clin Neurol 2016; 12: 274-81.

8. Saji E, Kawachi I, Nishizawa M. [Diagnosis and management of paraneoplastic neurological syndromes]. Brain Nerve 2010; 62: 319-30.

9. Bourque PR, Warman Chardon J, Bryanton M, Toupin M, Burns BF, Torres C. Neurolymphomatosis of the brachial plexus and its branches: case series and literature review. Can J Neurol Sci 2018; 45: 137-43.

10. Sideras PA, Matthews J, Sakib SM, Ofikwu F, Spektor V. Neurolym $\neg$ phomatosis of the peripheral nervous system: a case report and review of the literature. Clin Imaging 2016; 40: 1253-6. 